\title{
An equation simultaneously encodes the duality of the mind and the body
}

\author{
Zilin $\mathrm{Nie}^{1}$ and Yanming $\mathrm{Nie}^{2}$ \\ 1 Department of Infectious Diseases, Mayo Clinic, 200 First St. SW, Rochester, \\ MN 55905, USA \\ 2 College of Information Engineering, Northwest A \& F University, Yangling, \\ Shaanxi, China 712100 \\ Corresponding authors: nie.zilin@mayo.edu; yanmingnie@nwafu.edu.cn
}

Keywords: Mind-body problem; Mind-body coupling phenomenon; Entropic system; Entropic system equation; Law of entropic system; Self-organized criticality (SOC); Selforganized critical triggering factor (SOCTF); Conscious strength; Macrostates of consciousness; Self-organization of minds.

The mind-body problem is the central issue in both of philosophy and life science for several centuries. To date, there is still no conclusive theory to interpret the relation between the mind and the body, even just the mind alone. Here, we promote a novel model, a derived mathematic equation called the entropic system equation, to describe the innate characters of the mind and the mechanism of the mind-body coupling phenomenon. As the semi-open thermodynamic systems far from equilibrium, the living organisms could be logically considered as an entropic system. In the living organisms or the entropic systems, there also are three essential existing elements including mass, energy and information, in which the mind and the body are hypothetically coupled by free energy and entropic force.

\section{Introduction}

In philosophy, the answer to the mind-body problem is the key criteria to distinguish the materialism and the idealism (1). In idealism, the mind is the basic existence and the originating source of every subject in the universe. In materialism, the existent sequence between the mind and the body is reverse to the idealism. The endless and clueless debate scientifically is like the augment of the properties of wave and of particle in photon quantum mechanics, which finally is ended until the promotion of the wave- 
particle duality theory (2). There is not surprising that physicalism naturally stand on the side of materialism that supposedly mind is a physical phenomenon from the biochemical and biophysical processes. However, physicists also believe that there is no material, but energy and information. Up to date, both biochemists and biophysicists are unable to provide the conclusive answer theoretically and/or experimentally. The main obstacle might be what the relationship between thought and consciousness are in the mind. Moreover, it is still clueless what are the thought and the consciousness itself.

To provide the final answer to the mind-body problem, the first step has to understand the creation of the universe from the beginning. It seems to be a more and more common concept that the entropic nature begins from the creation to the end, if happened, of the universe. Thus, the initial universe was a symmetric vacuum state (the quantum vacuum as a singularity expanded exponentially the so-called inflation), and the symmetry of the universe gradually lost (3). It is known that asymmetry from the symmetry breaking creates entropy, an arrow for time of the universe (4). The pioneer in thermodynamics, Rudolf Clausius concluded two essential principles of entropy. First, the energy of the universe is constant. Secondly, the entropy of the universe tends to a maximum. Therefore, Arthur Eddington and other physicists, perhaps including the greatest Albert Einstein, believe the entropy always increasing is the highest principle among the law of Nature (5). The increase of the entropy results in the irreversible symmetry breaking of the natural processes leading to the asymmetry between past and future. The asymmetrical state in the universe absolutely is not even but stochastically fluctuating everywhere. The statistical mechanics pioneer, Ludwig Boltzmann hypothesized the fluctuating entropy as the physics thought experimental self-conscious body, so called Boltzmann brain, spontaneously and briefly formed in everywhere of the universe (6). In 1948, Claude Shannon promoted the pioneering concept of information entropy which could be possibly introduced into the time-arrow of the psychology and/or mind perception (7).

The entropy law holds the supreme position among the laws in both the non-living system and the living organisms. The asymmetry of molecules in water is also an initial force to generate life organism (8). In this article, the entropy system is hypothesized a unit of the essential structures which harbor the three elements of the mass, the energy and the information both in the non-living system and living organism. An entropy system with the constant consists of five variables, microstate, macrostate, free energy, entropic force and entropy itself. The self-organizations of the bio-entropic system are accomplished by the coordination of the mass, the energy and the information. Theoretically, the putative mechanism of the mind-body problem could be unveiled in the processes of the self-organization described with the entropic system equation, which simultaneously encodes the duality of the mind and the body. 


\section{Entropic system and law of the entropic system}

Every existence in the universe, from atom to universe itself, could be designed as an entropic system theoretically. An entropic system is composed of five basic elements, microstate $(\Omega)$, macrostate $(\Delta \mathrm{X})$, free energy $(\mathrm{Q})$ or temperature $(T)$, entropic force $\left(F_{\Delta X}\right)$ and entropy $(S)$ its own. In statistical mechanics, entropy value depends on the number of microstates in (9),

$$
S=\kappa \ln \Omega
$$

In the equation, $\mathrm{K}$ is Boltzmann constant. The thermodynamics entropy in the isolated thermodynamic system is determined by the average of free energy on the system's temperature (10),

$$
S=\frac{Q}{T}
$$

The entropic force mediates the entropic interaction acting on a system's macrostate (11), that is,

$$
F_{\Delta X}=\frac{T S}{\Delta \mathrm{X}}=\frac{Q}{\Delta \mathrm{X}}
$$

From above equations, we have,

$$
\begin{gathered}
\left(\frac{\Delta \mathrm{X}}{\ln \Omega}\right)\left(\frac{\mathrm{F} \Delta X}{T}\right)=\left(\frac{\Delta \mathrm{X}}{\ln \Omega}\right)\left(\frac{\mathrm{F} \Delta X}{Q}\right) S=K, \\
\text { or } \\
\left(\frac{\Delta \mathrm{X}}{\ln \Omega}\right)\left(\frac{\mathrm{F} \Delta X}{Q}\right)\left(\frac{\mathrm{S}}{\kappa}\right)=1
\end{gathered}
$$

Thus, as an exist of any entropic system, the ratio of macrostates and natural logarithm of microstates times the ratio of entropic force and temperature is equal to Boltzmann constant. Alternatively, the ratio of macrostates and natural logarithm of microstates times the ratio of entropic force and free energy, and continuously times thermodynamics entropy is equal to Boltzmann constant. The internal relationship of the elements of an entropic system and Boltzmann constant is named as the law of the entropic system. Theoretically, the law of the entropic system should be at the highest position in the universe and could be applicable from the system of elementary particles to black hole, as well as the universe itself. In the universal ideal gas, Boltzmann constant represents the constant value of the isolated entropic system. Therefore, we could name Boltzmann constant as the entropic system constant $\left(E_{C}\right)$. In an isolated entropic system, free energy $(Q)$ is a key player controlling the phenotype formation of the macrostates and the generation of the entropic forces, through the regulation of the 
diversity of the energy distribution (thermodynamics entropy) and the thermodynamic of the microstates (statistical mechanics entropy) under the control of the entropic system constant.

\section{Self-organization of an entropic fluid system}

In an entropic system, there are three absolute uncertain elements, microstate, free energy and entropy, and three relative certain elements, macrostate, entropic force and entropic system constant respectively. The putative mechanism of the self-organization is hypothetically controlled by the self-organized criticality (SOC) and self-organized critical triggering factor (SOCTF), such as one-dimensional $1 / f$ fractal noise, twodimensional $1 / \mathrm{f}$ fractal noise and three-dimensional $1 / \mathrm{f}$ fractal noise etc. (12). In fact, $\mathrm{SOC}$ is a macrostate of the system powerfully far from equilibrium. The self-organization could happen only after interacted with SOCTFs as in "the sandpile effect" (12).

In the isolated entropic system, there must be a constant from the integrations of the mass, the energy and the information. Boltzmann constant is a physical constant of fluid from the ratio of the constant of universal ideal gas to Avogadro's constant $\left(N_{A}\right)$ without counting the effects of particles. In the universe, there are three types of fluids, gas, liquid and solid with zero of flow speed, all of which should possess their fluid constants. In the Newton's liquid fluid, the constant could be approximately calculated by the Bernoulli equation (13),

$$
P+\frac{1}{2} \rho v 2+\rho g h=\mathrm{C}
$$

Where $P$ is the pressure at a certain point in the fluid, $v$ is the velocity of the fluid at that point, $\rho$ is the fluid density, $g$ is the acceleration of gravity, $h$ is the height at that point, and $C$ is a constant. In the isolated liquid fluid, the value of the Boltzmann constant, entropic system constant of universal ideal gas needs to be corrected as the entropic system constant $\left(E_{c}\right)$,

$$
E_{C}=\frac{C}{N A}
$$

From the equation 2.4, in the given isolated liquid fluid we will have,

$$
\left(\frac{\Delta \mathrm{X}}{\ln \Omega}\right)\left(\frac{\mathrm{F} \Delta X}{T}\right)=\left(\frac{\Delta \mathrm{X}}{\ln \Omega}\right)\left(\frac{\mathrm{F} \Delta X}{Q}\right) S=E_{C}
$$

In the open liquid fluid system far from equilibrium, the entropic system constant will be a function dynamically varying with the entropic elements generated internally or input externally,

$$
E_{C}=f\left[\left(\frac{\Delta X}{\ln \Omega}\right)\left(\frac{F_{\Delta \mathrm{X}}}{Q}\right) S\right]
$$


When an open system is far from equilibrium, the entropic system constant $\left(E_{c}\right)$ will be increased or decreased. The distant value $(\Delta \kappa)$ from equilibrium is equal to entropic system constant after removing Boltzmann constant in a universal ideal gas system,

$$
\Delta \kappa=E_{C}-\kappa
$$

The uncertainty in the thermodynamic system far from equilibrium, usually caused by the fluctuation of heat free energy, leads the increase of entropy via the thermodynamics of the microstates. The minimum energy principle in thermodynamic system forces the raised free energy into the dropping state through the heat dissipation, which leads to self-organization occurring locally to generate the certainty in the system. Alternatively, from the equation 1.7 we will have,

$$
\begin{gathered}
\left(\frac{\Delta X}{\ln \Omega}\right)\left(\frac{\mathrm{F}_{\Delta X}}{Q}\right)=\frac{E_{C}}{S} \\
\text { or } \\
\left(\frac{\Delta X}{\ln \Omega}\right)\left(\frac{\mathrm{F}_{\Delta X}}{Q}\right)\left(\frac{S}{E_{C}}\right)=1
\end{gathered}
$$

\section{Self-organizations of mass, energy and information in living systems}

Living organism belongs to a semi-open fluid far from equilibrium thermodynamics with the properties of the power-law fluids and the high potentials for the self-organizations through continuum mechanics. A typical example of the self-organization in the medium of water is hydrophobic force or entropic force driving lipid molecule to form the double layer membrane, which is believed as the prototype cell in living organism creation (14) (Figure 1).

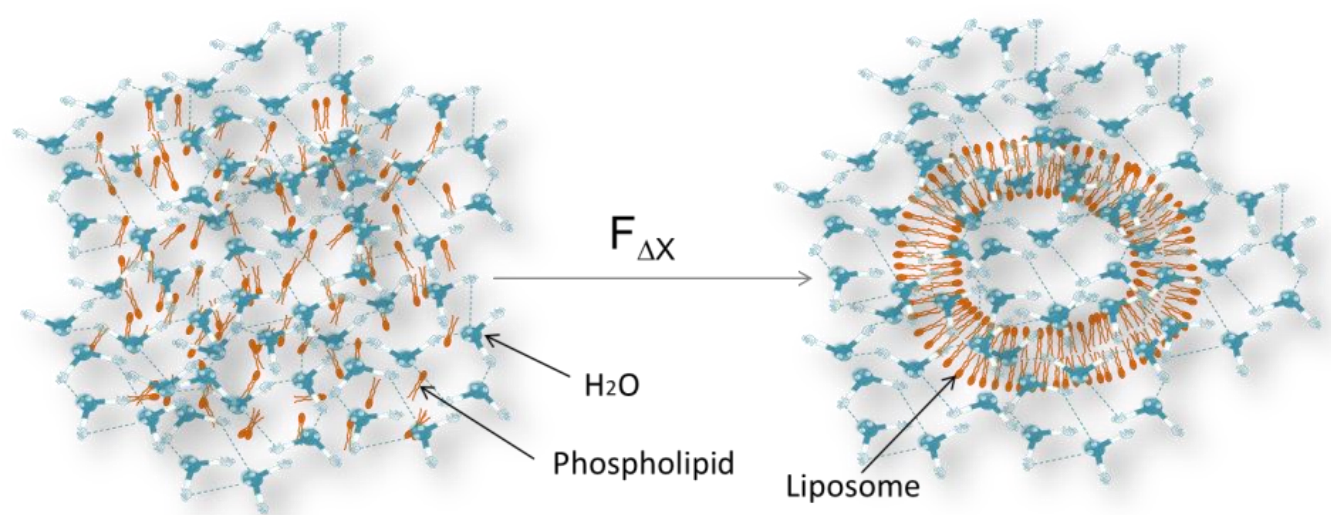

Figure 1: The schematic depiction for the entropic forces $\left(F_{\Delta^{x}}\right)$ driving the liposome formation, Phospholipid, water molecule and double layer structure of phospholipid are pointed by arrows. 
The water solution within the prototype cell belongs to a type of Newtonian fluid which is lacking the power to drive the division of the system. The self-organization of the entropic system are the thermal dynamic processes between the uncertain exists of the microstates and the relatively certain exists of macrostates driven by the free energy and the entropic forces under the guidance of the entropy and the entropic system constant. The power law distributions, either the mirror images and/or the concentrations of the organic chemicals, generate the states of the self-organized criticality (SOC) in liquid fluids leading to the assembly of the biological complexes or/and the syntheses of the large bio-molecules (15). Indeed, it is the entropic forces and the bio-enzymes mediating the molecular self-organization via the covalent bonds or/and the secondary bonds, such as hydrogen bond, ionic bond, ion-induced dipole forces, ion-dipole forces and van der Waals forces. In quantum mechanics, the formation of the chemical bonds, both the covalent bonds and the secondary bonds, relies on the entropic forces, the superposition probabilities of the sharing electron pair between the adjacent atoms. The large bio-molecule syntheses gradually increase the power laws of fluids and structurally fractalize the fluid system into the non-Newtonian scale-free networks. Nonetheless, the entropy and entropic force in the fractal networks are the key players in the self-organizations of the large molecules and the replication of the organisms.

The protocells are a self-organized spherical structure of the ordered lipid molecules, proposed as a first stepping-stone toward the origin of life (16). To maturate into cell which could actively replicate through fission or/and division, protocell should reach the macrostate of replication SOC during the period of revolution. Organism is a super entropic system cascading by variety of sub-entropic system arrays. The selforganization of each individual layer of entropic system only happens after reaching its SOC and simultaneously existing responsive SOCTFs such as enzymes, cytokines and ligands etc. in a manner of domino-like cycles. The formation of continuous SOCs and SOCTFs is the key step for the life revolution of the prototype maturation. The appearance of DNA replication, RNA transcription and protein translation, so called biological central dogma of self-organization cycles, terminates the living organism from the nonliving system. In fact, organisms are types of power-law fluids believed to be original from Newtonian fluid of water solutions in the sea (17). The self-organization processes could happen in the cells with the SOCs via power-law principle which is insufficient in protocell. ATP molecules produced in the metabolism and the catalytic enzymes accelerate the self-organization via the increase and decrease of the SOC values respectively. In the biological central dogma, the power law distributions of the microstates, the ATP molecules and the enzymes create the SOCs and the SOCTFs for DNA replication, RNA transcription and protein translation. In short, the macrostate's transitions of the DNA replication, RNA transcription and protein translation are the three key steps in the cell replication cycles (Figure. 2): 


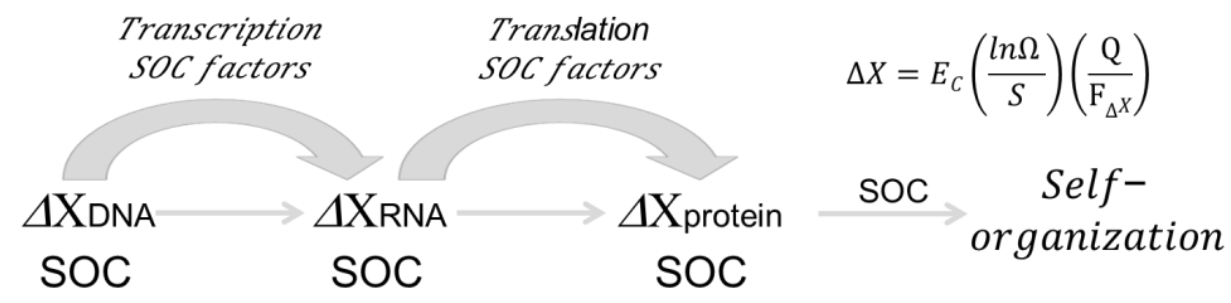

Figure 2: The schematic depiction of the dynamic flow of the macrostates in the biological central dogma. SOC: self-organized criticality. The macrostate transitions occur as domino's effect in the dogma.

The self-organizations of the macrostate's transition through the DNA replication, RNA transcription and protein translation sequentially integrate living organisms as the entire networks of an entropic system with the continuous arrays of the entropic elements including the mass, the energy and the information. In the continuous entropic arrays, the systemic uncertainty is absolutely principle while the systemic certainty is relatively temporal processes in life processes.

\subsection{Self-organization of the biological structure}

The self-organization of the biological structures in the advanced living organisms has to be mainly accomplished through the so-called central dogma, DNA replication, RNA transcription and protein translation in cell cycle. The large biomolecules further form the sub-cellular organs via the self-assembly mechanism by flowing the law of the entropic system. The biological significances of the sub-cellular organ structures are the power law-enhanced distributions of the cellular masses, the cellular energies and the cellular information.

\subsection{Self-organization of the biological function}

The biological functions in the advanced living organisms are self-organized through the formation of the sub-cellular organs. In the self-organization processes, the differentiated cells are gathered as organ systems in symmetrical and/or supersymmetrical manners. Based on histological anatomy, there are at least 11 major organ structural systems, that is, nervous system, endocrine system, cardiovascular system, lymphatic system, respiratory system, digestive system, urinary system, integumentary system, skeletal system, muscular system and reproductive system. In post genomic era, the systems are divided as genomics, epigenomics, lipidomics, proteomics, glycomics, transcriptomics, metabolomics, nutrigenomics, foodomics etc.. The systems are functionally complementary each other to control the self-organization of the human body. 


\subsection{Self-organization of the biological information}

The information of the self-organization is always synchronized as the forces of free energy driving the reorganization of the microstates and the macrostates. In quantum mechanics, besides the dually of particle and wave, there is also individual information generated in particle movement in wave manners. In any system which consists of element particles, atoms and molecules, the law of the entropic system always terminates the interactions of material, energy and information. In the biological system, the mind is a kind of information generated during the self-organization of biomolecule mass and metabolism of biological energy. According to Shannon's information theory of entropy, the conscious strength $(\Psi)$ in cellular entropic system could be estimated as,

$$
\Psi=-\sum_{i=1}^{n}\left(\frac{E_{\mathrm{c} i}}{s_{i}}\right) \log \left(\frac{E_{\mathrm{c} i}}{S_{i}}\right)
$$

There are also three groups of variables in the entropic system equation, the microstate and macrostate, the free energy and entropic force, the entropic system constant and entropy, which correspond to material or mass (structural body), energy (coupling force) and biological information "consciousness" respectively (Figure.3).

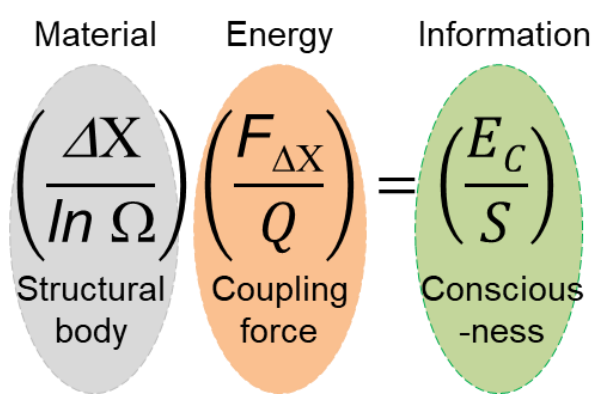

Figure 3: The equation depicting the entropic system equation for the mind coupled body phenomenon. The representatives of the variable parameters are the same as the descripted as above.

The consciousness is the common phenotype of macrostate existing in every thermodynamic system in the universe. Nonetheless, the self-organizations in the brain neural networks include both cellular structures and the spiritual minds. The strength of the mind $\left(\Psi_{\text {cell }}\right)$ in every individual cell could be determined by the ration of the entropic system constant and the thermodynamic entropy. The conscious concept above might be helpful to uncover the mysteries of the cellular behaviors, such as the plasticity, the migration, the proliferation, the differentiation, the reprogramming and the cell death etc.. In the human body, the differentiated cells from the different tissues or organs possess the differences of the consciousness and mind because of the sizes, the masses, the energies and the entropies, which determine the intelligence quotients (IQs) for the coordination of their behaviors (Figure. 4). 
A

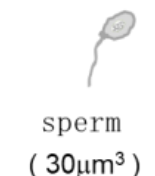

(ax)

Oocyte $\left(4 \times 10^{6} \mu m^{3}\right)$
Red cell

$\left(100 \mu m^{3}\right)$

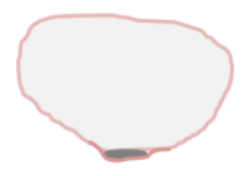

Fat cell $\left(6 \times 10^{5} \mu m^{3}\right)$

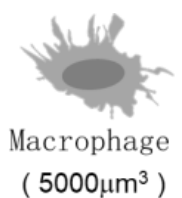

$\left(5000 \mu \mathrm{m}^{3}\right)$

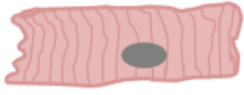

Cardiomyocyte $\left(15000 \mu \mathrm{m}^{3}\right)$

B

$$
\begin{aligned}
\Psi_{\text {Cell }}=-\sum_{i=1}^{n}\left(\frac{E c i}{S i}\right) \log \left(\frac{E c i}{S i}\right) & =-\sum_{i=1}^{n}\left(\frac{\Delta X}{\ln \Omega}\right)\left(\frac{\mathrm{F}_{\Delta} X}{Q}\right) \log \left(\frac{\Delta X}{\ln \Omega}\right)\left(\frac{\mathrm{F}_{\Delta} X}{Q}\right) \\
\Psi_{\text {Organ }} & =\sum_{i=1}^{n=\infty} \Psi_{\text {iCell }}
\end{aligned}
$$

Figure 4: The schematic depiction for the conscious strengths of cells and organs. A: The differences of the cells from the various organs or the tissues in the sizes and the morphologies. B: The equations depicting the conscious strengths of cells and organs.

\section{Self-organization in brain neural networks}

The consciousness is the essential cradleland and shelter of mind in our brain neural networks. Probably, the mind is only formed in advanced living organisms which possess the maturated neural networks derived from the terminally differentiated neurons. However, consciousness is a general feature in the universal existences, especially in various fluids with free energy fluctuation. However, the total entropic system constant $\left(E_{c i}\right)$ of the brain neural networks have to include the entropic system constants from the bodies of the neurons, the axons and dendrites of the networks, water solution fluids of cerebrospinal fluid (CSF) and the steam inside head etc..

\subsection{Self-organization of the brain neural networks}

The self-organization of the brain neural networks includes the neuron proliferation, neuron differentiation and neural network plasticity which is hallmarked with fractal characters in structure, energy and information. The neural networks in the brain consists neuron bodies, axons, dendrites and synapses etc. (Figure 5). 

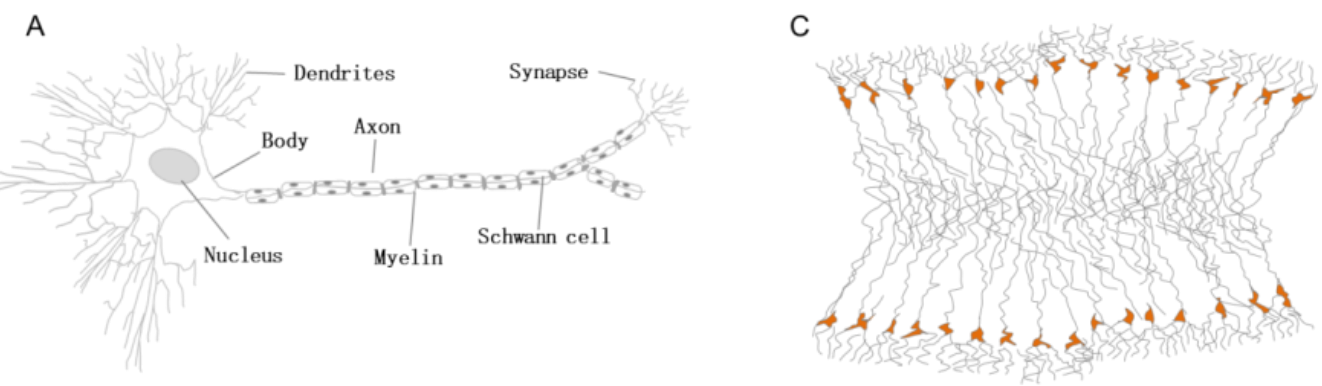

$\Psi_{\text {Neuron }}=\sum_{i=1}^{n} \Psi_{\text {Body }}+\Psi_{\text {Axon }}+\Psi_{\text {Dendrites }}+\Psi_{\text {Synapse }}+\cdots$

D $\Psi_{\text {Neuralnetworks }}=\sum_{i=1}^{n=\infty} \Psi_{\text {iNeuron }}$

Figure 5: The schematic and the equational depictions for the conscious strengths of the neurons and the neural networks. A. The representative of the neuron. B. The equational depiction for the conscious strengths of the neuron. C. The schematic depiction for the neural networks. D. The equational depiction for the conscious strength of the neural networks.

The consciousness is also the essential media in the learning-memory processes in the neural networks. Dr. Eric Kendall, the recipient of the 2000 Nobel Prize in Physiology or Medicine, promoted the model to describe the mechanism occurred in the learning and memory (18). In learning period, the stimulation signals are transmitted in the neural networks by action potentials, which trigger the intracellular signaling to form the short memory. However, the continuous transduction of the stimulation signals could sequentially initiate the gene expression of the memory relevant proteins to induce the plasticity of the neural networks to create long term memory (Figure. 6).

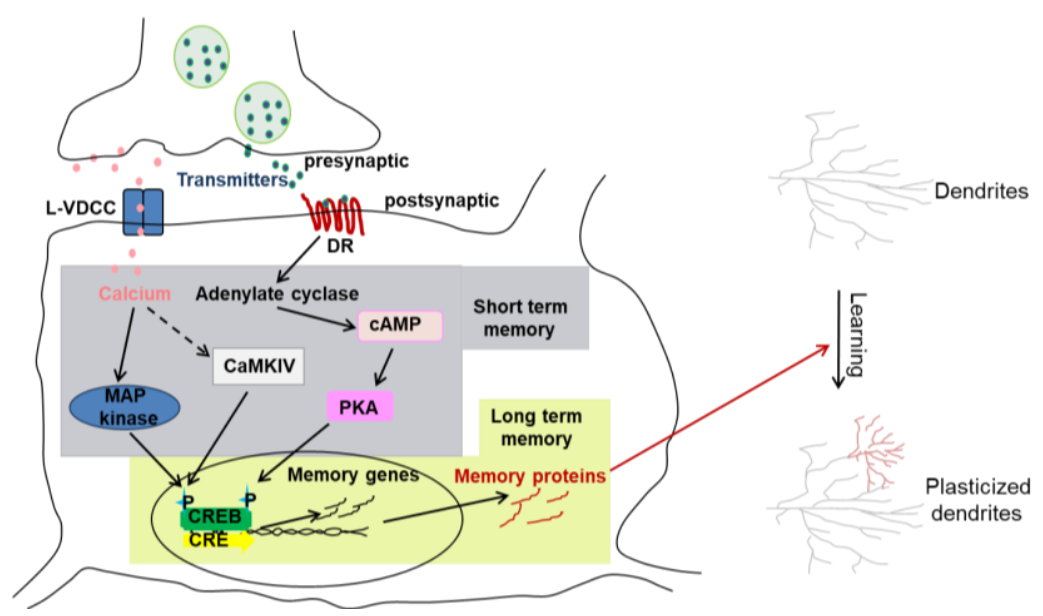

Figure 6: The schematic depiction for the biological cascades of the learning and the memory. L-VDCC: L-Type Voltage-Dependent Calcium Channel. DR: Dopamine receptor. cAMP: Cyclic adenosine monophosphate. CaMKIV: Calcium/calmodulin-dependent protein kinase IV. PKA: Protein kinase A. CREB: CAMP response element-binding protein. CRE: cAMP response element. 


\subsection{Self-organization of the conscious minds}

The consciousness is one of the three macroscopic states, matter, energy and information in the entropic systems of the neural networks. Basing on the equation of the entropic system, the conscious strength of the living organisms, from single cells to human beings, depends on the ratios of the entropy and the entropic system constant. Although the self-organizations of the biological structures occurred in the neural networks might relate to the self-organization of the minds to create memory and thought, the minds in brain must be the highest macrostate in the entropic system of the conscious information converged from the body's cells. The biological structures, the cells and the tissue matrices, are the thermodynamic systems far from the equilibrium. Thus, the conscious strengths of them should fluctuate as the wave spectrum dynamically varying from time to time. The conscious SOCs, formed via discrete Fourier transform (DFT) from the body's spectral entropies $(19,20)$, initiate every interface transferring of the macrostates by the informatic noises of the internal body and outside environment. The mind is formed the power spectral entropies of the brain neural networks over the rest of the body even "second brain" in the abdominal cavity (21). Recently, the SOC theory has been emergent for understanding how the conscious mind created in neural networks (22). In a hypothetical model, the SOC might also play the fundamental roles in the neural plasticity and the mind creation via the power law of the spectral entropies. Although the spectral entropies quantify the power spectral density (PSD) of an EEG signals, the spectra formed via DFT of the neural networks are not the real conscious mind (Figure 7).

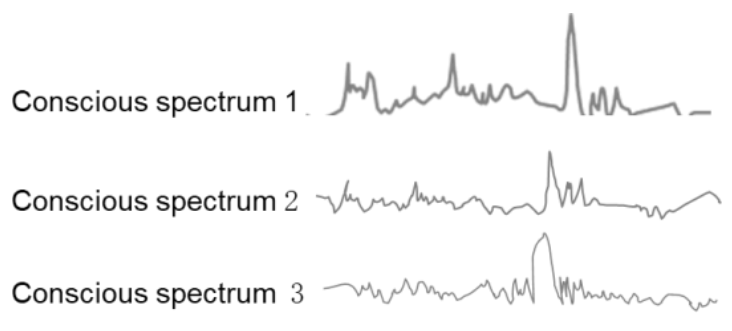

Discrete Fourier transform

(DFT)

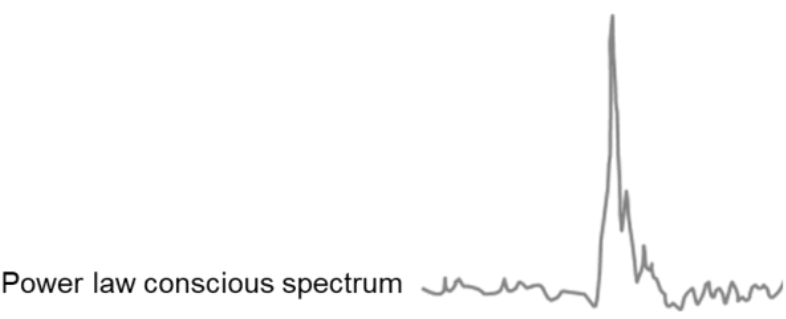

Figure 7: The schematic depiction for the generation of the power law of the spectral entropies. 
The spectral entropies represent the uncertainty of the nervous pulses of action potentials oscillating in the neural networks. It is believed the action potentials might play roles in the free energy production, the gene expression and the signal transduction in the neurons, the plasticity of the synapses in the neural networks and probably the entropic noises triggering the self-organization of the consciousness and mind. In fact, all the conscious minds belong to the informatic phenotypes of the macrostates in the brain neural networks. In the conscious self-organization processes, to have a mind, to keep a mind and even to erase a mind, all of them are sort of the interface transferring of the different types of the informatic macrostates. Based on the SOC theory, the self-organization of the conscious mind has to be triggered by the critical factors, such as the $1 / f$ conscious noises from the action potentials induced by the stimulations from the external environment and/or the internal body including thinking. Therefore, the interface transferring of the SOCs is the key step for the macroscopic states of perceptions, sensations, feelings, thoughts, memories, fantasies, emotions and instincts (Figure 8).

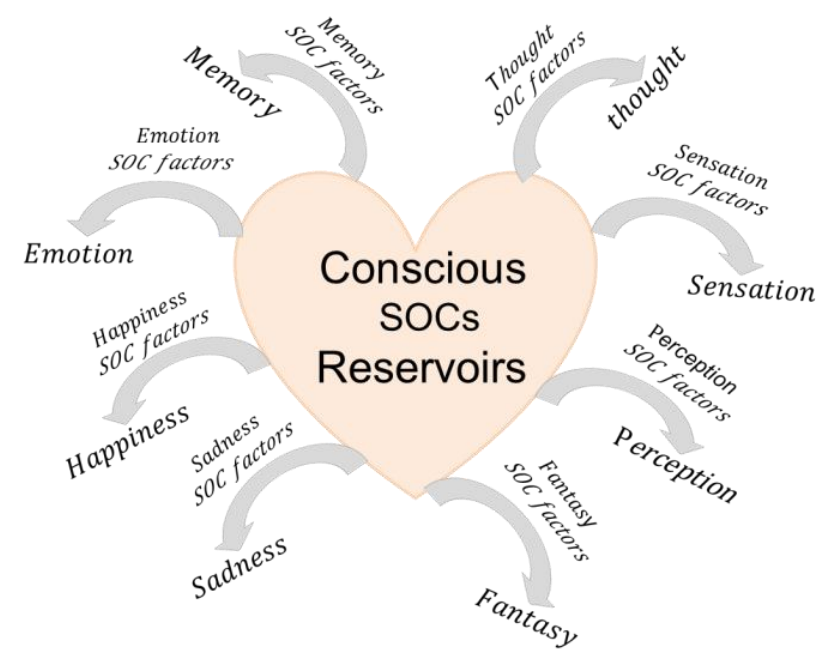

Figure 8: The schematic depiction for the conscious interface transition of the macrostate's SOCs via the responsive SOC factors.

\section{Mind and body coupling in human body}

Through the logic thinking, the mind in the brain could control all the body to coordinate the physiological activities the behaviors. Comparing to the other parts of the human body, the human head is the most prominent in the biological structures, the energy supplement and the information production. Structurally, there are three major parts in the head, the brain neural networks, the additional cerebrospinal fluid (CSF) with the steams and the skull bone (Figure 9). 


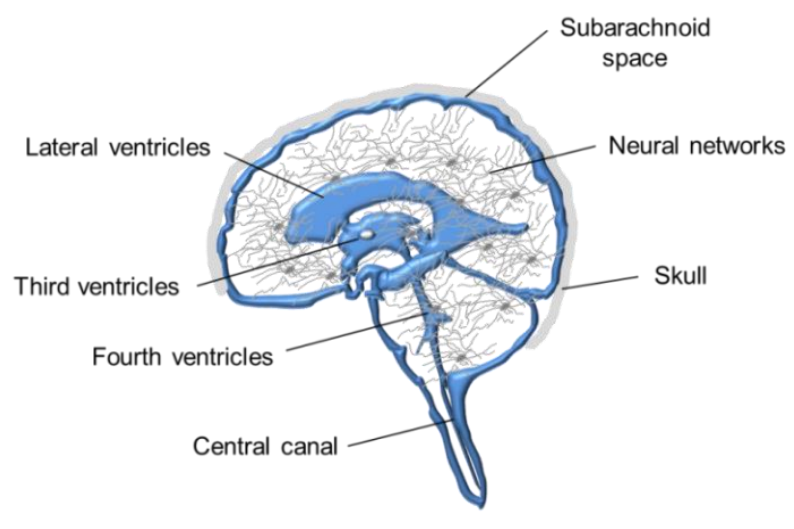

Figure 9: The schematic depiction for the neural networks, CSF (cerebrospinal fluid) and skull bone.

The fractal characters of the axons and dendrites in the neural networks are the continuous scale-free networks of the traces of the entropic system constants. There are also three types of entropies in the brain neural networks, the entropy of the free energy distributions (thermodynamic entropy), the entropy of the microstate disorders (statistical entropy) and the entropy of the information certainty (information entropy). The duality between the structure and the information is a natural character in the entropic system, which is coupled by entropic force and free energy. The consciousness is a macrostate in the informatic entropic system of the brain neural networks. The SOCs in the conscious entropic system are created by the power spectral entropies from all the neural networks via a similar DFT mechanism. The duality of mind and body is also a natural feature in human beings. The mind is composed of variety of information macrostates harbored in the brain neural networks including perceptions, sensations, feelings, thoughts, memories, and fantasies etc.. Each single cell in human body possesses the consciousness governing its own behavior. However, the conscious strengths of the body's cells are distributed in a power-law manner (23). The mind and the body are coupled through the conscious strength of the brain neural networks which dominates all other parts of the body. According Landauer's principle, to erase one bit of information requires a minimum amount of energy calculated by the following equation (24),

$$
E=\kappa T \operatorname{In} 2
$$

Information could be supposedly converted into energy via quantum mechanics. To command the body, the mind in the brain neural networks must be strong enough to power through the erasure of that in other tissues or organs. In other words, mind and body are the information and the mass co-existing in the same entropic system of the human body. The brain neural networks create the mind which controls the behaviors of the body in power law manner (Figure 10). 

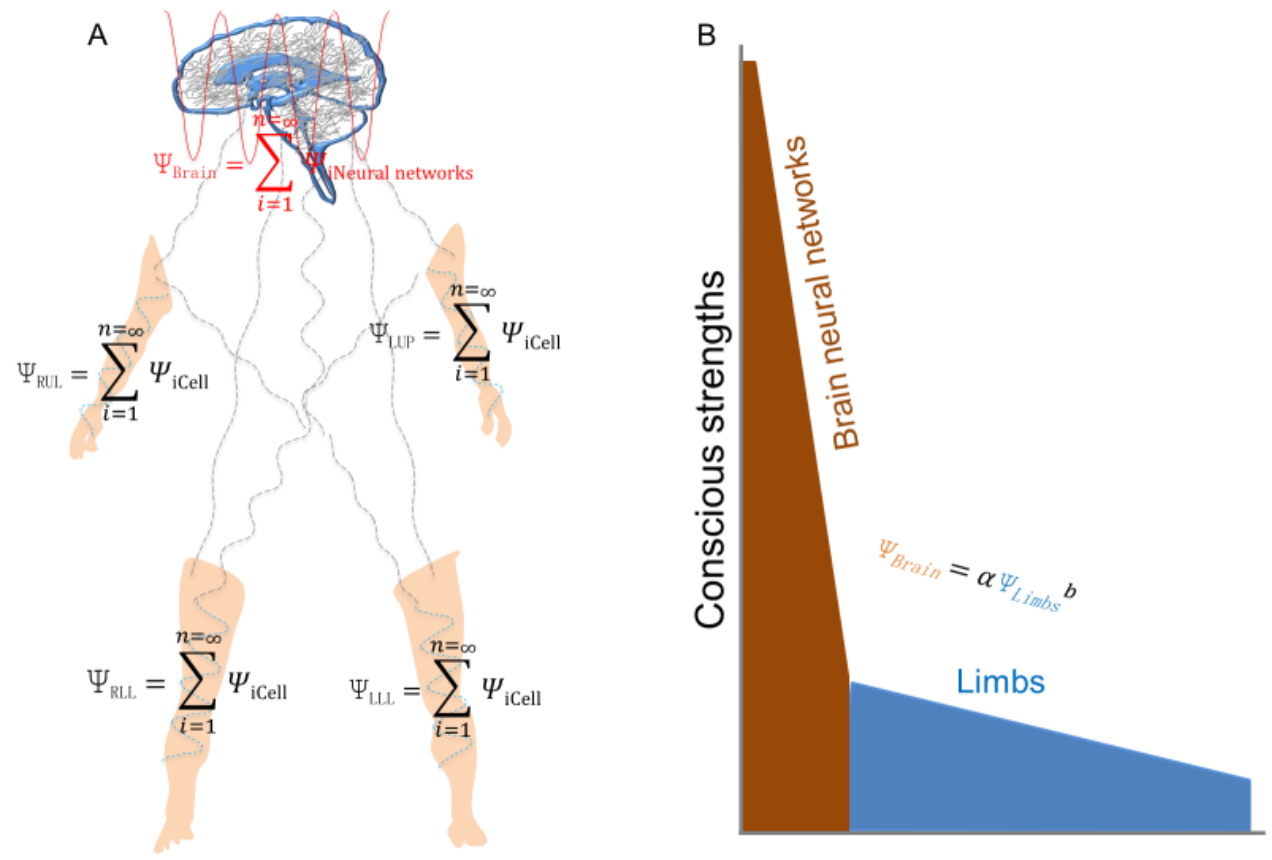

Figure 10: The schematic depiction for the hypothesized power law model of the mind over the consciousness of the human body. $\Psi$ : conscious strength, Ec: entropic system constant, S: thermodynamic entropy, B: brain, $\mathrm{H}$ : heart, RUL: right upper limb, LUL: left upper limb, RLL: right lower limb, LLL: left lower limb.

\section{Concluding remarks}

Indeed. it is a so long debate between the idealism and the materialism on the mind and body problem. The core of the issue is the internal relationships among matter, energy and information. There is no doubt that most modern scientists are standing on the side of materialism, in a line with physicalism believing "everything is physical" or "nothing over and above the physical". However, the putative concept of mass-energyinformation equivalence principle might provide us the novel insight into the mind and body problem, in which mass represents the energy and information (25). Nonetheless, there are still far from enough to appease idealism theoretically and/or experimentally. To shed light on the mind-body problem, a theoretical model of entropic system is promoted here to unveil the mystery of the relationship between mind and body.

The principle of the entropy is thought to be the highest in the interpretation of the natural phenomenon. In the entropic system, all biological molecules, cells and even human bodies are just the different levels of entropic units in which the macrostate transitions occur along with the fluctuations of the certain amount of energy and information though the thermodynamic mechanisms of the microstates. The state and the consequence of the subject in time-space axis absolutely rely on the interaction of the microstate, macrostate, free energy, entropic force, entropy and entropic system 
constant. As one of Einstein's most famous quotes "God does not play dice with the universe", the law of the entropic system in the universe determines the fates of the any subject, from the element particles, Brownian motion molecules and even planets. From a physics point of view, the spontaneous symmetry breaking provides the mass and the energy to the element particles via Higgs mechanism. Meanwhile, the spontaneous symmetry breaking creates the disorders leading to the generation of the entropic system. The three essential elements of the mass, the energy and the information arrays cascade all the layers from quantum elements to the universe itself. In the equilibrium system at any layer, the relation and the interaction of the three essential elements should obey the law of the entropic system. In living organisms, especially in the brain of the advanced animals such as human beings, consist of gas fluids, liquid fluids and the solids (e.g., the bones which could be considered as a fluid with the zero speed of movement). There are also three types of the entropic systems in living organism, biological structure entropic system (mass), free energy entropic system (energy) and Shannon entropic system (information). In the entropic systems, the SOCs might be the common properties in statistical entropic system (structure), thermodynamic entropic system (energy) and Shannon entropic system (information) for the initiations of the self-organizations. In human mind, perceptions, sensations, feelings, thoughts, memories, fantasies, emotions and instincts, even the feelings of happy and sad etc., all of them are belong to information macrostates in the information entropic system of the brain neural networks. Truly, the minds are just the feelings of the macrostate strengths sharply powering from one to another. Therefore, the interface transferring of the macrostates of information must be the key to have and keep a mind in the brain. The interface transferring of a mind is a process of self-organization in the information entropic system, which requires the SOCs of the entropic system and SOCFs of the entropic noises. In the human brain, the information entropic noises could come from the entropic spectra generated in the action potential pulses fluctuating in the neural networks. The brain neural networks are plentiful with mitochondria, consuming about $40 \%$ oxygens of all human body. The energy supply system promotes the brain neural networks a conscious unique to create the powerful minds.

An entropic system with the constant is composed of five essential elements, microstate, macrostate, free energy, entropic force and entropy itself. In the system, all elements crossly depend on and interact on each other. In living organism, all phenotypes including the systemic states of function and information of the biological structures, as well as the biological structures themselves, could be designed as the macrostates. While entropic force works on the macrostates, entropy commutes between the system and the environment in the dissipative model promoted by Nobel laureate, Dr. llya Prigogine (26). Entropic systems function as the self-organization units in human body. When the macrostates bearing the SOCs meet the corresponding the self-organized critical factors of the bio-noises, the interface transferring may occur 
during the physiologically associated macrostates. Under the state of the sleep, the brain neural networks might be away from the conscious SOCs. In awakening, the brain neural networks must stay on the conscious SOCs in order to transfer the states of the conscious macrostates of the minds including variety of the emotions and the thoughts. Indeed, the entropic forces in the continuous arrays of the brain neural networks might be essential for the communication from person to person who shares the common macrostates. All in all, the entropic force is a kind of information force from the entropy of the system. In nature, the importance of entropic forces is suspicious to be underestimated. At the end, various natural forces are entropic forces on specific macrostates. The gravity, so called entropic gravity, is also hypothesized as an entropic force (27). To interpret the mind-body problem in a biological entropic system, it is necessary to fully understand the bridge-role of free energy and entropic force which couple the information of the mind and the behavior of the body. The entropic forces are types of information powers, perhaps equally acting on the mass, the free energy and the information in the brain neural networks and the rest of all body. Otherwise, human body could be functioned as a super entropic system, in which the mind and the body are coupled by entropic forces (Figure. 2 and 3). In short, it is difficult for us to solve the mind-body problem based on either genomic biology or post-genomic biology despite the advances of bioinformatics and systemic biology. To develop approaches to explore the mathematical and biological entropic system may be an emergent promising to answer what is the mind when debating on the mind-body problem.

Finally, there is no question that the entropic system model might provide the novel promise for resolving the mind-body problem. However, the entropic system model also promotes more questions about the conscious minds. For example, as in an entire entropic system, both of cerebrospinal fluid (CSF) and skull just protect the brain and the neural networks? May the entropic system be the best model for the systems biology, so called as the entropic systems biology? Truly, the modern molecular biology and energy metabolism have already provided deep insights into the microstates, the macrostates, the free energies and the entropies. Again, it is still very difficult to calculate the entropic system constants of the tissue cells, the organs, the systems and all the body. Nonetheless, it is strongly emergent with the developments of the mathematical and physical approaches to define the SOCs, SOCFs and the entropic system constants of the neurons and the human brain neural networks.

\section{References}

1. Skirry, J (2016). "Rene Descartes: The Mind-Body Distinction". Internet Encyclopedia of Philosophy. ISSN 2161-0002. 
2. Einstein A, Infeld L (1938). The Evolution of Physics: The Growth of Ideas from Early Concepts to Relativity and Quanta. Cambridge University Press.

3. Anderson, P.W (1972). "More is Different". Science. 177 (4047): 393-396. doi:10.1126/science.177.4047.393.

4. Weinert, $F$ (2005). The scientist as philosopher: philosophical consequences of great scientific discoveries. Springer. p. 143. ISBN 978-3-540-21374-1., Chapter 4, p. 143

5. Clausius, RJE (1879). The mechanical theory of heat (Translated by Walter R. Browne), Literary Licensing, LLC, Aug 7, 2014 - 390 pages.

6. Carroll, S (2008). "Richard Feynman on Boltzmann Brains". Retrieved 24 June 2019.

7. Shannon, CE (1948). "A Mathematical Theory of Communication". Bell System Technical Journal. 27 (3): 379-423. doi:10.1002/j.1538-7305.

8. Garay, A, Keszthelyi, L, Demeter, I et al (1974). Origin of asymmetry in biomolecules. Nature 250, 332-333.

9. Zemansky, MW (1968). Heat and Thermodynamics, 5th ed.: An Intermediate Textbook New York: McGraw-Hill, p. 215

10. Neumann RM (1980). "Entropic approach to Brownian movement". American Journal of Physics. 48 (5): 354-357.

11. Feynman, R (1970). The Feynman Lectures on Physics Vol I. Addison Wesley Longman. ISBN 978-0-201-02115-8.

12. Bak, P., Tang, C. and Wiesenfeld, K (1987). "Self-organized criticality: an explanation of 1/f noise". Physical Review Letters. 59 (4): 381-384.

13. Batchelor, G. K (2000). An Introduction to Fluid Dynamics. Cambridge: Cambridge University Press. ISBN 978-0-521-66396-0.

14. Marsh, P (2012). Thermodynamics of Phospholipid Self-Assembly. Biophys J. 102(5): 1079-1087. doi: 10.1016/j.bpj.2012.01.049

15. Seybold, H. J., Carmona, H. A., Herrmann, H. J., and Andrade, J.S. (2019) Selforganization in purely viscous non-Newtonian turbulence. Phys. Rev. Fluids 4, 064604

16. Chen, A., Walde, P (2010). From Self-Assembled Vesicles to Protocells. Cold Spring Harb Perspect Biol. 2 (7): a002170.

17. G. W. Scott Blair et al., J. Phys. Chem. (1939). The flow of cream through narrow glass tubes.43 (7) 853-864

18. Kandel, E.R (2007). In Search of Memory: The Emergence of a New Science of Mind, New York: W. W. Norton \& Company, ISBN 978-0-393-32937-7.

19. Gilbert, S (1994). "Wavelets". American Scientist. 82 (3): 250-255. JSTOR 29775194

20. Domenico, M.D, Biamonte, J (2016). Spectral entropies as information-theoretic tools for complex network comparison. Phys. Rev. X 6, 041062 
21. Yates, D (2017). Organizing the second brain. Nat Rev Neurosci 18, 387

22. Bennett, C.H (2003). "Notes on Landauer's principle, Reversible Computation and Maxwell's Demon", Studies in History and Philosophy of Modern Physics, 34 (3): 501-510

23. Hou L, Kwok J.T (2018). Power Law in Sparsified Deep Neural Networks. arXiv:1805.01891

24. Hubler, A, Belkin, A, Bezryadin, A (2015). "Noise induced phase transition between maximum entropy production structures and minimum entropy production structures?" Complexity. 20 (3): 8-11. doi:10.1002/cplx.21639.

25. Vopsona M.M (2019). The mass-energy-information equivalence principle. AIP Advances 9, 095206; doi:10.1063/1.5123794

26. Prigogine I (1978). Time, structure, and fluctuations. Science 1;201(4358):77785.

27. Verlinde, E.P (2011). "On the origin of gravity and the laws of newton". JHEP. (4): 29. doi:10.1007/JHEP04(2011)029 